\title{
CARACTERIZAÇÃO DAS PLANTAS PRODUZIDAS NO HORTO MUNICIPAL DA BARREIRINHA - CURITIBA / PR
}

\author{
Daniela Biondi ${ }^{1}$, Luciana Leal $^{2}$ \\ (recebido em 14.03.2008 e aceito para publicação em 02.06.2008)
}

\section{RESUMO}

Esta pesquisa teve como objetivo analisar as características das plantas produzidas no Horto Municipal da Barreirinha, na cidade de Curitiba/PR, quanto aos aspectos relativos ao ecossistema local e danos a saúde humana. De uma lista de 165 plantas, 43,03\% foram exóticas, $6,67 \%$ foram consideradas invasoras no Brasil, 3,03\% declaradas como invasoras no Estado do Paraná e 9,09\% apresentaram algum princípio tóxico ou alérgicos. Recomenda-se a adoção desta análise para estabelecer critérios para a seleção e a produção de mudas de espécies adequadas para as áreas públicas da cidade de Curitiba.

Palavras-chave: viveiro municipal, plantas ornamentais, espécies nativas, espécies tóxicas, espécies invasoras.

\footnotetext{
${ }^{1}$ Engenheira Florestal, Dra., Professora Depto. Ciências Florestais, Universidade Federal do Paraná - UFPR, Curitiba. Bolsista Produtividade em Pesquisa - CNPq (dbiondi@ufpr.br)

2 Engenheira Florestal, M.Sc. Doutoranda em Engenharia Florestal - UFPR. Bolsista do CNPq. (luciana_paisagem@yahoo.com.br)
} 


\title{
CHARACTERIZATION OF THE PLANTS PRODUCED AT THE HORTO MUNICIPAL DA BARREIRINHA - CURITIBA / PR
}

\begin{abstract}
The aim of this research was to analyze the characteristics of the plants produced at the Horto Municipal da Barreirinha of Curitiba/PR in relation to damages to local ecosystems and human being health. From a list of 165 plants, 43.03\% were exotic species, $6.67 \%$ were considered as invasive plants in Brazil, 3.03\% declared as invasive plants in the State of Paraná and $9.09 \%$ presented some toxic or allergic principles. The adoption of this analysis is recommended to establish criteria for the selection and the seedlings production of adequate species for public areas of the city of Curitiba.
\end{abstract}

Key-words: municipal nursery, ornamental plants, native plants, poisonous plants, invasive plants. 


\section{INTRODUÇÃO}

A maior parte da vegetação que compõe a arborização urbana de uma cidade é produzida em viveiros municipais. Conforme Gonçalves et al. (2004), a produção de mudas para uso próprio em grandes cidades, na maioria das vezes, é plenamente justificável para plantas ornamentais e arborização urbana, pois a aquisição destas de terceiros pode ser bastante onerosa, em virtude da quantidade requerida.

Além do uso na arborização urbana, a vegetação produzida em viveiros municipais pode servir de suporte para campanhas e atividades de educação ambiental nas escolas, recuperação de áreas degradadas e revitalização de áreas de proteção, como as encostas e as nascentes. Gonçalves et al. (2004), avaliando viveiros municipais no estado de Minas Gerais, constataram que a maioria dos municípios que possuem viveiros, produzem mudas para arborização urbana, recuperação de áreas degradadas e matas ciliares, sendo estas destinadas principalmente para doação.

Devido a grande diversidade de funções que esta vegetação desempenha nos ambientes urbanos, fazem-se necessários critérios rígidos na seleção de espécies, tanto com relação ao ecossistema local, como em relação ao público que convive nestes espaços. Embora o desempenho da arborização urbana seja reflexo da forma com que se faz o seu planejamento, isto também pode ser um produto do que se produz nos viveiros.

Atualmente existe uma grande preocupação com a biodiversidade nas áreas urbanas e isto faz com que o responsável pelo viveiro aumente e diversifique o número de espécies produzidas. De-Knegt et al. (2005) afirmam que a vegetação variada distribuída nas áreas verdes e arborização viária no município de Belo Horizonte - MG permite que vários animais silvestres, dentre eles os quirópteros, ainda encontrem na cidade um ambiente adequado à sobrevivência.

No ecossistema urbano, quanto maior a diversidade de espécies vegetais, maior a possibilidade de instalação definitiva de uma fauna mais diversificada. De acordo com Biondi e Kischlat (2006), quanto maior o número de espécies presentes no ecossistema, maior é a sua capacidade de resistir às variações e de absorver impactos negativos, como a poluição, e as adversidades climáticas, e menores são as possibilidades do surgimento de pragas e doenças que afetam a fauna e a flora.

A preocupação com a diversificação de espécies, muitas vezes, faz com que não haja tempo suficiente para realizar pesquisas sobre as espécies, ocorrendo produções de espécies indesejáveis para o ambiente e o homem - é o caso das plantas tóxicas e as plantas exóticas invasoras.

A árvore urbana deverá, via de regra, gerar conforto à população e não causar problemas, mesmo em casos isolados. Características de árvores, tais como, a presença de 
espinhos, dificulta o manejo e pode provocar acidentes; princípios alérgicos, principalmente com pólen, manifestam-se nas pessoas de diferentes formas; plantas tóxicas têm seu efeito assegurado quando contraída as partes nocivas (SANTOS; TEIXEIRA, 2001). A ausência de princípios tóxicos é um dos fatores mais importantes na seleção de espécies para o meio urbano (CESP, 1988; BIONDI; ALTHAUS, 2005).

Reis et al. (2003) consideram importante a biodiversidade na arborização urbana desde que antes de efetuar a introdução de uma nova espécie no meio, seja realizado um histórico da mesma, verificando se ela já apresentou características de invasora em outros países e se possui potencial para se tornar uma nova espécie invasora. Considera-se importante aplicar o princípio da precaução no tocante à introdução de novas espécies no país. Isto é, uma espécie nova só deve ser introduzida se já houver o conhecimento de seu comportamento ecológico e não houver potencialidade para contaminar ecossistemas naturais. Segundo Reichard e White (2001), a maioria das plantas invasoras foi introduzida para uso na horticultura por viveiros, jardins botânicos ou por pessoas.

As características das espécies que irão compor a cobertura vegetal pública de uma cidade devem ser criteriosamente analisadas, antes mesmo de serem produzidas em viveiros. Sendo assim, o objetivo deste trabalho foi analisar as características das plantas produzidas no Horto Municipal da Barreirinha - Curitiba/PR quanto aos aspectos relativos a danos à saúde humana e ao ecossistema local.

\section{MATERIAIS E MÉTODOS}

Este trabalho adotou como estudo de caso as espécies produzidas no Horto Municipal da Barreirinha, da Secretaria Municipal do Meio Ambiente, da Prefeitura Municipal de Curitiba. Este viveiro fica localizado no bairro Barreirinha, na cidade de Curitiba e foi criado na década de 60. É responsável pela pesquisa e produção de mudas de árvores para a arborização pública (ruas e áreas verdes), e também de mudas de arbustos ornamentais e frutíferas silvestres, para recuperação de áreas degradadas e adensamento de bosques (PREFEITURA, 2008). Neste Horto é realizada, tanto a fase de produção de mudas em viveiro de semeadura (15 mil mudas/ano), como a fase de condução e rustificação em viveiro de espera, no solo ou em embalagens (91700 mudas). A área destinada à produção de mudas para arborização viária é de $80 \mathrm{mil} \mathrm{m}^{2}$ e a área construída (incluindo escritório, refeitório, almoxarifado, banheiro, garagem, casa de repicagem e depósito de substrato) é de $904 \mathrm{~m}^{2}$ (LEAL, 2007).

$\mathrm{Na}$ listagem de espécies utilizada foram consideradas, tanto as espécies atualmente produzidas no viveiro de semeadura, como também as espécies armazenadas no viveiro de espera, até o ano de 2007. 
Todas as espécies foram classificadas quanto à sua procedência: nativa do Brasil ou exótica. Para as espécies nativas foram identificadas aquelas com ocorrência nos ecossistemas que originalmente formavam a cobertura vegetal da cidade de Curitiba: a Floresta Ombrófila Mista (Floresta com Araucária) e a Estepe Gramíneo-Lenhosa (Campos). Também foram classificadas quanto à sua forma de vida: arbórea, arbustiva, herbácea ou trepadeira.

As espécies foram analisadas quanto à presença de características inadequadas ao uso público, como a presença de princípios alérgicos e/ou tóxicos. A identificação das plantas tóxicas e/ou com princípios alérgicos foi feita segundo Albuquerque (1980), Frohne e Pfänder (1983), Blackwell (1990), Henderson (2001), Fiocruz (2001), Lorenzi e Mattos (2002), Agra et al. (2007) e Jardim de Flores (2007).

Outra característica analisada foi quanto à classificação das espécies como plantas invasoras no Brasil, segundo Hórus (2007), e no Estado do Paraná, conforme Paraná (2007). Também foram levantadas as espécies que são consideradas invasoras em outros países, pois segundo Biondi e Pedrosa-Macedo (2008), estes dados podem indicar as espécies potenciais invasoras também no Brasil. Isto vai depender das condições facilitadoras do ambiente e do seu uso indiscriminado. Este levantamento foi realizado através de um check-list (INVASIVE PLANTS DATABASE, 2007), que fornece as espécies e os países em que são consideradas invasoras. Também foi consultada a lista de espécies consideradas invasoras na África, apresentada por Henderson (2001), e nos Estados Unidos, por Swearingen (2007). Outras fontes consultadas foram o The Global Compendium of Weeds (GCW, 2007), que só forneceu a informação se a espécie é invasora, sem a discriminação do local, e Bingelli et al. (1998), que citam as espécies consideradas invasoras nos trópicos e subtrópicos.

\section{RESULTADOS E DISCUSSÃO}

\section{Aspectos relativos ao ecossistema local}

De um total de 165 espécies consideradas, 135 são árvores (81, 82\%), 28 são arbustos (16,97\%) e 2 trepadeiras (1,21\%), correspondentes a 57 famílias e 132 gêneros (Tab. 1). As famílias com maior número de espécies são: Fabaceae (37), Myrtaceae (13) e Bignoniaceae (8).

O Horto Municipal produz, principalmente, espécies nativas no Brasil: 94 (56,97\%). Das espécies produzidas destinadas à arborização de ruas, também a maior parte é de plantas nativas no Brasil: 18 (64,28\%). Atualmente, o aumento na produção de espécies nativas é um aspecto importante, pois segundo Biondi e Althaus (2005), tanto em Curitiba como em outras cidades brasileiras, o uso de espécies nativas na arborização de ruas é 
limitado. Barroso et al. (2007) afirmam que a utilização de plantas nativas ameaçadas de extinção na ornamentação de jardins, parques, praças, ruas, residências manterá um banco genético muito maior que aquele de instituições de pesquisa, jardins botânicos, bancos de germoplasma, etc.

Porém, verifica-se que ainda é pequeno o número de espécies nativas nos ecossistemas ocorrentes na cidade de Curitiba, encontrando-se nesta pesquisa o total de 44 espécies (26,67\%). Segundo Ziller e Zalba (2007), o termo espécie nativa refere-se a espécies dentro dos limites de sua distribuição natural em termos evolutivos, ou seja, a limites ambientais e não políticos. Espécies movidas de um ecossistema a outro, dentro de um país, são tão exóticas quanto espécies não-nacionais e também apresentam um alto potencial de risco e dano.

Um aspecto negativo encontrado nesta avaliação é que neste viveiro há a produção de mudas de espécies consideradas invasoras. Um percentual de 6,67\% das espécies produzidas no Horto é invasora no Brasil (Acacia longifolia, Acacia mearnsii, Acacia podalyriaefolia, Casuarina equisetifolia, Coffea arabica, Eryobotrya japonica, Hovenia dulcis, Morus nigra, Psidium guajava, Schizolobium parahyba e Spartium junceum) e 3,03\%, no Estado do Paraná (Acacia mearnsii, Eryobotrya japonica, Hovenia dulcis, Morus nigra e Psidium guajava).

De acordo com a Portaria no 95, de 22 de maio de 2007 (PARANÁ, 2007), que reconhece a Lista Oficial de Espécies Exóticas Invasoras para o Estado do Paraná, os imóveis públicos nos quais for constatada a presença de espécies exóticas invasoras, devem, obrigatoriamente, proceder à sua erradicação ou controle, para evitar a contaminação biológica. Assim, estas espécies não deverão ser mais produzidas neste viveiro. Para Mack et al. (2000) e Burt et al. (2007), a prevenção de iniciais introduções, ao invés de subseqüente controle ou erradicação, é a forma mais efetiva de evitar gastos associados com combate de plantas invasoras. Ziller e Zalba (2007) afirmam que entre as ações para evitar e resolver problemas relacionados a espécies invasoras está o não cultivo de plantas ornamentais exóticas invasoras e a não utilização de espécies exóticas em projetos de restauração ambiental.

As espécies que foram consideradas invasoras em outros países relacionados neste trabalho chegam a ter $29,09 \%$ de ocorrência, sendo que $22,42 \%$ são espécies que não são consideradas invasoras no Brasil.

\section{Aspectos relativos à saúde humana}

Quanto à presença de princípios tóxicos, 15 plantas (9,09\%) apresentam princípio tóxico em alguma parte da planta. Dentre as plantas tóxicas encontradas podem ser destacadas Nerium oleander L. (espirradeira) e Spartium junceum L. (giesta), em que todas 
as partes da planta são consideradas tóxicas. Nerium oleander, segundo Blackwell (1990), é considerado como o arbusto ornamental mais tóxico.

Este fator é relevante a ser considerado, pois estas mudas não poderão ser indicadas para determinados usos, como a doação para escolas e creches ou o plantio em praças, que são locais com livre acesso de crianças, com as quais, segundo Santos e Teixeira (2001), ocorrem a maioria dos casos de intoxicação com plantas. Conforme Lucas (1997), o envenenamento de crianças por plantas tóxicas ocorre como resultado destas comerem frutos, flores ou folhas de plantas, atraídas pela sua cor ou cheiro.

Biondi et al. (2008), em trabalho sobre as espécies vegetais encontradas nas escolas estaduais da cidade de Curitiba, verificaram que, de um total de 170 espécies amostradas, $26(15,29 \%)$ apresentaram algum princípio tóxico e/ou alérgico em alguma parte da planta. Provavelmente muitas das mudas destas plantas consideradas tóxicas foram adquiridas no Horto da Barreirinha.

Cavalcanti et al. (2003), em análise das espécies produzidas, destinadas ao Horto Florestal Lauro Pires Xavier, na cidade de Campina Grande - PB, constataram que das 22 espécies vegetais produzidas, 13\% apresentam algum tipo de princípio tóxico. Estes autores também consideraram preocupante a presença de plantas tóxicas, já que grande parte dessas plantas é implantada em praças, parques e creches da cidade, onde há uma grande circulação de crianças. 
Tabela 1 - Plantas produzidas no Horto Municipal da Barreirinha - Curitiba/PR quanto à sua adequação ao ecossistema local e à saúde humana

\begin{tabular}{|c|c|c|c|c|c|c|c|c|}
\hline № & NOME CIENTÍFICO & NOME POPULAR & FAMÍLIA & F. V. & P. & TOXICAS & INVASORAS & Uso \\
\hline 1 & Abelia x grandiflora (Rovelli ex André) Rehder & Abélia & Caprifoliaceae & $\mathrm{Ar}$ & $E$ & & GCW & $\mathrm{AV}$ \\
\hline 2 & Acacia longifolia (Andrews) Willd. & Acácia- trinervis & Fabaceae & A & $E$ & & IP, NZ, E, P, As, Ar, Br, T & $A V$ \\
\hline 3 & Acacia mearnsii De Wild. & Acácia- negra & Fabaceae & A & $\mathrm{E}$ & & $\mathrm{IP}, \mathrm{P}, \mathrm{EUA}, \mathrm{Ar}, \mathrm{Br}, \mathrm{T}, \mathrm{As}$ & $\mathrm{AV}$ \\
\hline 4 & Acacia podalyriaefolia A. Cunn. ex G. Don & Acácia-mimosa & Fabaceae & A & E & & As, $\mathrm{T}, \mathrm{Br}, \mathrm{GCW}$ & AV \\
\hline 5 & Acer negundo L. & Acer & Aceraceae & A & E & & G, E, Hu, U, Po, Ar, T, A, P, GCW & $\mathrm{R}, \mathrm{AV}$ \\
\hline 6 & Acer palmatum Thunb. & Acer-palmatum & Aceraceae & A & $\mathrm{E}$ & & & AV \\
\hline 7 & Aleurites fordii Hemsl. & Tungue & Euphorbiaceae & A & $\mathrm{E}$ & $\begin{array}{l}\text { sementes } \\
\text { outras partes }\end{array}$ & e IC, T, GCW & $\mathrm{R}, \mathrm{AV}$ \\
\hline 8 & Allamanda puberula A. DC. & Alamanda & Apocynaceae & $\mathrm{Ar}$ & $\mathrm{N}$ & seiva & & $A \vee, A B$ \\
\hline 9 & Allophyllus edulis (St.-Hil.) Radlk & Vacum & Sapindaceae & A & NC & & & $\mathrm{R}, \mathrm{AV}$ \\
\hline 10 & Anadenanthera colubrina (Vell.) Brenan & Monjoleiro & Fabaceae & A & $\mathrm{N}$ & & & $\mathrm{R}, \mathrm{AV}$ \\
\hline 11 & Annona cacans Warm. & Araticum-cagão & Annonaceae & A & $\mathrm{N}$ & & & $\mathrm{AV}, \mathrm{AB}$ \\
\hline 12 & Araucaria angustifolia (Bertol.) Kuntze & Araucaria & Araucariaceae & A & NC & & GCW, T & $\mathrm{AV}, \mathrm{AB}$ \\
\hline 13 & Ardisia crenata Sims & Ardísia & Myrsinaceae & $\mathrm{Ar}$ & $E$ & & As, IP, Ma, T, GCW, EUA & $\mathrm{AV}$ \\
\hline 14 & Balfourodendron riedelianum (Engl.) Engl. & Pau-marfim & Rutaceae & A & N & & & $A \vee, A B$ \\
\hline 15 & Bauhinia forficata Link & Pata-de-vaca & Fabaceae & A & NC & & & $\mathrm{AV}, \mathrm{AB}$ \\
\hline 16 & Bauhinia variegata $\mathrm{L}$. & Pata-de-vaca & Fabaceae & A & E & & As, IP, IC, GCW, EUA & $\mathrm{R}, \mathrm{AV}$ \\
\hline 17 & Bougainvillea glabra Choisy & três-marias & Nyctaginaceae & $\operatorname{Ar}$ & NC & & & $\mathrm{AV}, \mathrm{AB}$ \\
\hline 18 & Bougainvillea spectabilis Willd. & três-marias & Nyctaginaceae & $\mathrm{Ar}$ & $\mathrm{E}$ & & & $A V$ \\
\hline 19 & Brunfelsia uniflora (Pohl) D. Don & Manacá & Solanaceae & $\operatorname{Ar}$ & NC & & & $\mathrm{AV}, \mathrm{AB}$ \\
\hline 20 & Butia eriospatha (Mart. ex Drude) Becc. & Butiá & Arecaceae & A & N & & & $A \vee, A B$ \\
\hline 21 & Buxus sempervirens $\mathrm{L}$. & Buxinho & Buxaceae & $\mathrm{Ar}$ & $\mathrm{E}$ & folhas & & $\mathrm{AV}$ \\
\hline 22 & Cabralea canjerana (Vell.) Mart. & Canjarana & Meliaceae & A & N & & & $A V, A B$ \\
\hline 23 & Caesalpinia echinata Lam. & Pau-brasil & Fabaceae & A & N & & & $A V, A B$ \\
\hline 24 & Caesalpinia leiostachya (Benth.) Ducke & Pau-ferro & Fabaceae & A & $\mathrm{N}$ & & & $\mathrm{R}, \mathrm{AV}, \mathrm{AB}$ \\
\hline 25 & Caesalpinia peltophoroides Benth. & Sibipiruna & Fabaceae & A & N & & & $R, A V, A B$ \\
\hline 26 & Caesalpinia spinosa (Molina) Kuntze & Pau-brasil (falso) & Fabaceae & $A$ & E & & & $\mathrm{AV}$ \\
\hline 27 & Caliandra brevipes Benth. & Caliandra-rosa & Fabaceae & $\operatorname{Ar}$ & $\mathrm{N}$ & & & $A \vee, A B$ \\
\hline
\end{tabular}


28 Caliandra tweedii Benth.

29 Callistemon citrinus (Curtis) Skeels

30 Camelia japonica L.

31 Campomanesia guazumaefolia (Camb.) Berg

32 Campomanesia xanthocarpa O. Berg

33 Capsicodendron dinisii (Schwacke) Occhioni

34 Carya illioinensis (Wang.) K. Koch

35 Casearia decandra Jacq.

36 Casearia sylvestris Sw.

37 Cassia ferruginea (Schraeder) Schrader ex DC.

38 Cassia laevigata Willd.

39 Cassia leptophylla Vogel

40 Castanea sativa Mill.

41 Casuarina equisetifolia L.

42 Cedrela fissilis Vell.

43 Centrolobium tomentosum Guillemin ex Benth.

44 Chamaecyparis obtusa (Siebold \& Zucc.) Endl.

45 Chamaerops humilis $\mathrm{L}$.

46 Chorisia speciosa A. St.-Hil.

47 Citharexylum myrianthum Cham.

48 Citrus limon (L.) Burm.

49 Clethra scabra Pers.

50 Coffea arabica L.

51 Copaifera langsdorfii Desf.

52 Cordia superba Cham.

53 Cotoneaster franchetti Bois

54 Cryptomeria japonica (Thunb. ex L. F.) D. Don

55 Cuninnghamia lanceolata (Lamb.) Hooker

56 Cupania vernalis Cambess.

57 Cupressus lusitanica Mill.

\begin{tabular}{|c|c|c|c|c|c|}
\hline Caliandra-vermelha & Fabaceae & $\operatorname{Ar}$ & $\mathrm{N}$ & & \\
\hline Calistemon & Myrtaceae & $\operatorname{Ar}$ & $E$ & & $\mathrm{~T}$ \\
\hline Camélia & Theaceae & A & $\mathrm{E}$ & & \\
\hline Sete-capotes & Myrtaceae & $A$ & NC & & \\
\hline Guabiroba & Myrtaceae & $A$ & $\mathrm{NC}$ & & \\
\hline Pimenteira & Canellaceae & A & $\mathrm{NC}$ & & \\
\hline Noz-pecã & Junglandaceae & $A$ & $E$ & & \\
\hline Guaçatunga & Flaucourtiaceae & $A$ & $\mathrm{NC}$ & & \\
\hline Cafezinho-bravo & Flacourtiaceae & A & $\mathrm{NC}$ & & \\
\hline Cássia-ferruginea & Fabaceae & $A$ & $\mathrm{~N}$ & & \\
\hline Cássia-levigata & Fabaceae & $A$ & $\mathrm{~N}$ & & \\
\hline Cássia-fastuosa & Fabaceae & A & $\mathrm{N}$ & & \\
\hline Castanha-portuguesa & Fagaceae & $A$ & $\mathrm{E}$ & & GCW, T \\
\hline Casuarina & Casuarinaceae & $A$ & $E$ & flores (irritação) & As, $\mathrm{Br}$ \\
\hline Cedro-rosa & Meliaceae & A & $\mathrm{NC}$ & & \\
\hline Araribá & Fabaceae & $A$ & $\mathrm{~N}$ & & \\
\hline Cedro-europeu & Cupressaceae & $A$ & $E$ & & \\
\hline Palma-leque & Arecaceae & $A$ & $E$ & & \\
\hline Paineira & Bombacaceae & $A$ & $\mathrm{~N}$ & & \\
\hline Jacataúva & Verbenaceae & $A$ & $\mathrm{~N}$ & & \\
\hline Limão-rosa & Rutaceae & $A$ & $\mathrm{E}$ & & \\
\hline Carne-de-vaca & Clethraceae & $A$ & $\mathrm{NC}$ & & \\
\hline Café & Rubiaceae & $\operatorname{Ar}$ & $E$ & & $I P, I C, I G, T$ \\
\hline Pau-óleo & Fabaceae & $A$ & $\mathrm{~N}$ & & \\
\hline Cordia & Boraginaceae & A & $\mathrm{N}$ & & \\
\hline Cotoneaster & Rosaceae & Ar & $\mathrm{E}$ & $\begin{array}{ll}\text { frutos } & \text { (em } \\
\text { quantidade) }\end{array}$ & \\
\hline Criptoméria & Taxodiaceae & $A$ & $E$ & & EUA \\
\hline Cuningamia & Taxodiaceae & A & $E$ & & EUA \\
\hline Cuvatã & Sapindaceae & $A$ & NC & & \\
\hline Cipreste & Cupressaceae & A & $\mathrm{E}$ & & \\
\hline
\end{tabular}

AV,

AV

AV

$A V, A B$

$A V, A B$

$A V, A B$

AV

$A V, A B$

$A V, A B$

$A V, A B$

$A V, A B$

$R, A V, A B$

AV

AV

$A V, A B$

$A V, A B$

AV

AV

$A V, A B$

$A V, A B$

AV

$A V, A B$

$A V, A B$

$A V, A B$

$A V, A B$

AV

AV

AV

$A V, A B$

AV 
58 Cupressus sempervirens $\mathrm{L}$.

59 Cybistax antisyphilitica (Mart.) Mart.

60 Dalbergia nigra (Vell.) Allemao ex Benth.

61 Diospyros kaki L.

62 Enterolobium contortisiliquum (Vell.) Morong

63 Eriobotrya japonica (Thunb.) Lindl.

64 Erythrina crista-galli L.

65 Erythrina falcata Benth.

66 Erythrina speciosa Andrews

67 Erythroxylum argentinum O. E. Schulz

68 Eucalyptus cinerea F. Muell. ex Benth.

69 Eugenia involucrata DC.

70 Eugenia pyriformis Cambess.

71 Eugenia uniflora L.

72 Euterpe edulis Mart.

73 Ficus insipida Willd.

74 Gardenia jasminoides J. Ellis

75 Gochnatia polymorpha (Less.) Cabrera

76 Grevillea banksii $\mathrm{R}$. $\mathrm{Br}$.

77 Hibiscus mutabilis $\mathrm{L}$.

78 Hibiscus rosa-sinensis $\mathrm{L}$.

79 Holocalyx balansae Micheli

80 Hovenia dulcis Thunb.

81 Hydrangea macrophylla (Thunb.) Ser.

82 Hypericum calycinum $\mathrm{L}$.

83 Ilex paraguariensis A. St.-Hil.

84 Inga marginata Willd.

85 Jacaranda mimosaefolia

86 Jacaranda puberula Cham.

87 Jasminum mesnyi Hance

$\begin{array}{llll}\text { Cedro-charuto } & \text { Cupressaceae } & \mathrm{A} & \mathrm{E} \\ \text { lpê-verde } & \text { Bignoniaceae } & \mathrm{A} & \mathrm{N} \\ \text { Dalbergia } & \text { Fabaceae } & \mathrm{A} & \mathrm{N} \\ \text { Caqui } & \text { Ebenaceae } & \mathrm{A} & \mathrm{E} \\ \text { Timboril } & \text { Fabaceae } & \mathrm{A} & \mathrm{N} \\ \text { Nêspera } & \text { Rosaceae } & \mathrm{A} & \mathrm{E} \\ \text { Corticeira-do-banhado } & \text { Fabaceae } & \mathrm{A} & \mathrm{NC} \\ \text { Corticeira-da-serra } & \text { Fabaceae } & \mathrm{A} & \mathrm{NC} \\ \text { Suína } & \text { Fabaceae } & \mathrm{Ar} & \mathrm{N} \\ \text { Marmeleiro/cocão } & \text { Erythroxylaceae } & \mathrm{A} & \mathrm{N} \\ \text { Eucalipto-prateado } & \text { Myrtaceae } & \mathrm{A} & \mathrm{E} \\ \text { Cereja } & \text { Myrtaceae } & \mathrm{A} & \mathrm{NC} \\ \text { Uvaia } & \text { Myrtaceae } & \mathrm{A} & \mathrm{N} \\ \text { Pitanga } & \text { Myrtaceae } & \mathrm{A} & \mathrm{NC} \\ \text { Palmito } & \text { Arecaceae } & \mathrm{A} & \mathrm{N} \\ \text { Figueira } & \text { Moraceae } & \mathrm{A} & \mathrm{N} \\ \text { Jasmim-branco } & \text { Rubiaceae } & \mathrm{Ar} & \mathrm{E} \\ \text { Cambará } & \text { Asteraceae } & \mathrm{A} & \mathrm{NC} \\ \text { Grevílea-anã } & \text { Proteaceae } & \mathrm{Ar} & \mathrm{E} \\ \text { Abutilon } & \text { Malvaceae } & \mathrm{Ar} & \mathrm{E} \\ \text { Hibisco } & \text { Malvaceae } & \mathrm{Ar} & \mathrm{E} \\ \text { Alecrim } & \text { Fabaceae } & \mathrm{A} & \mathrm{N} \\ \text { Uva-do-japão } & \text { Rhamnaceae } & \mathrm{A} & \mathrm{E} \\ \text { Hortência } & \text { Hydrangeaceae } & \mathrm{Ar} & \mathrm{E} \\ \text { Hipérico } & \text { Clusiaceae } & \mathrm{Ar} & \mathrm{E} \\ \text { Erva-mate } & \text { Aquifoliaceae } & \mathrm{A} & \mathrm{NC} \\ \text { Ingá } & \text { Fabaceae } & \mathrm{A} & \mathrm{N} \\ \text { Jacarandá-mimoso } & \text { Bignoniaceae } & \mathrm{A} & \mathrm{E} \\ \text { Caroba } & \text { Bignoniaceae } & \mathrm{A} & \mathrm{NC} \\ \text { Jasmim-amarelo } & \text { Oleaceae } & \mathrm{Ar} & \mathrm{E}\end{array}$

AV

$A V, A B$

$A V, A B$

AV

$A V, A B$

As, Ar, Br, GCW, EUA, NZ, T

$A V, A B$

$A V, A B$

$A V, A B$

$A V, A B$

AV

$A V, A B$

$A V, A B$

$A V, A B$

As, GCW, IC, IP, Ma, T AV, AB

$A V, A B$

AV

$\mathrm{AV}, \mathrm{AB}$

GCW, IP, EUA, T

AV

AV

GCW, IG

AV

$A V, A B$

$\mathrm{Ar}, \mathrm{Br}, \mathrm{IP}, \mathrm{T}$

AV

AV

GCW, T

$A V, A B$

$A V, A B$

$\mathrm{R}, \mathrm{AV}$

$A V, A B$

AV 
88 Koelreuteria paniculata Laxm.

89 Lafoensia pacari A. St.-Hil.

90 Lagerstroemia indica L.

91 Lamanonia ternata Vell.

92 Liquidambar styraciflua L.

93 Liriodendron tulipifera $\mathrm{L}$.

94 Lonchocarpus muehlbergianus Hassl.

95 Luehea divaricata Mart.

96 Magnolia grandiflora L.

97 Magnolia liliflora Desr.

98 Malvaviscus arboreus Cav.

99 Matayba elaeagnoides Radlk.

100 Maytenus ilicifolia Reissek

101 Michelia champaca L.

102 Mimosa flocculosa Benth.

103 Mimosa scabrella Benth.

104 Morus nigra L.

105 Myrceugenia euosma (Berg.) Legrand.

106 Murraya paniculata (L.) Jack

107 Myrcia hatschbachii D. Legrand

108 Myrciaria tenella (DC.) Berg.

109 Myrciaria trunciflora O. Berg

110 Nerium oleander L.

111 Ocotea porosa (Nees \& C. Mart.) Barroso

112 Ocotea puberula (Rich.) Nees

113 Parapiptadenia rigida Benth. (Brenan)

114 Passiflora edulis Sims

115 Peltophorum dubium (Spreng.) Taub.

116 Persea americana Mill.

117 Phoenix canariensis Chabaud

\begin{tabular}{|c|c|c|c|c|}
\hline Koleutéria & Sapindaceae & A & $E$ & \\
\hline Dedaleiro & Lithraceae & A & NC & \\
\hline Extremosa & Lithraceae & A & $E$ & casca \\
\hline Guapererê & Cunoniaceae & A & NC & \\
\hline Liquidambar & Hamamelidaceae & $A$ & E & \\
\hline Liriodendro & Magnoliaceae & A & $E$ & \\
\hline Lonchocarpus & Fabaceae & A & NC & \\
\hline Açoita-cavalo & Tiliaceae & A & NC & \\
\hline Magnólia & Magnoliaceae & A & $E$ & \\
\hline Magnólia-roxa & Magnoliaceae & $\mathrm{Ar}$ & $E$ & \\
\hline Malvavisco & Malvaceae & $\mathrm{Ar}$ & $E$ & \\
\hline Miguel-pintado & Sapindaceae & A & NC & \\
\hline Espinheira-santa & Celastraceae & A & NC & \\
\hline Magnólia-amarela & Magnoliaceae & A & $E$ & \\
\hline Bracatinga-branca & Fabaceae & A & $\mathrm{N}$ & \\
\hline Bracatinga & Fabaceae & A & NC & \\
\hline Amora & Moraceae & A & $E$ & \\
\hline Guamirim & Myrtaceae & A & NC & \\
\hline Murta & Rutaceae & A & $E$ & \\
\hline Caingá & Myrtaceae & A & NC & \\
\hline Cambuí & Myrtaceae & A & NC & \\
\hline Jaboticaba & Myrtaceae & A & NC & \\
\hline Espirradeira & Apocynaceae & $\operatorname{Ar}$ & $E$ & toda a planta \\
\hline Imbuia & Lauraceae & A & NC & \\
\hline Canela & Lauraceae & A & NC & \\
\hline Angico & Fabaceae & A & $\mathrm{N}$ & \\
\hline Maracujá & Passifloraceae & $\mathrm{T}$ & $\mathrm{N}$ & \\
\hline Canafístula & Fabaceae & A & $\mathrm{N}$ & \\
\hline Abacate & Lauraceae & A & $E$ & \\
\hline Fênix-canariense & Arecaceae & A & $\mathrm{E}$ & \\
\hline
\end{tabular}




\section{Phoenix roebilinii O'Brien}

119 Pithecolobium incuriale (Vell.) Benth.

120 Platanus occidentalis L.

121 Podocarpus lambertii Klotzsch ex Endl.

122 Populus alba L.

123 Populus nigra L.

124 Prunus sellowii Koehne

125 Prunus serrulata Lindl.

126 Psidium cattleyanum Sabine

127 Psidium guajava L.

128 Punica granatum L.

129 Pyracantha coccinea M. Roem.

130 Quercus robur L.

131 Rapanea ferruginea (Ruiz \& Pav.) Mez

132 Rhododendron simsii Planch.

133 Rhus succedanea L.

134 Robinia pseudoacacia L.

135 Rollinia silvatica (A. St.-Hil.) Martius

136 Roupala brasiliensis Klotzsch

137 Roystonea oleracea (Jacq.) O. F. Cook

138 Salix babylonica $\mathrm{L}$.

139 Sambucus australis Cham. \& Schltdl.

140 Schinus molle L.

141 Schinus terebinthifolius Radd

142 Schizolobium parahyba (Vell.) S.F. Blake

143 Sebastiania commersoniana (Bail.) L.B. Sm. 8

Fênix
Barbatimão
Plátano
Pinheiro-bravo
Populus-branco
Populus/álamo
Pessegueiro-bravo
Cereja-do-japão
Araçá
Goiaba
Romã
Piracanta
Carvalho-europe
Capororoca
Azaléia
Rus
Robínia
Araticum
Carvalho-brasileiro
Palmeira-imperia
Chorão
Sabugueiro
Aroeira-chorão
Guapuruvu
Branquilho

$\begin{array}{lll}\text { Arecaceae } & \text { A } & \text { E } \\ \text { Fabaceae } & \text { A } & \text { N } \\ \text { Platanaceae } & \text { A } & \text { E } \\ \text { Podocarpaceae } & \text { A } & \text { N } \\ \text { Salicaceae } & \text { A } & \text { E } \\ \text { Salicaceae } & \text { A } & \text { E } \\ \text { Rosaceae } & \text { A } & \text { N } \\ \text { Rosaceae } & \text { A } & \text { E } \\ \text { Myrtaceae } & \text { A } & \text { N } \\ & & \\ \text { Myrtaceae } & \text { A } & \text { N } \\ \text { Punicaceae } & \text { A } & \text { E } \\ \text { Rosaceae } & \text { Ar } & \text { E } \\ \text { Fagaceae } & \text { A } & \text { E } \\ \text { Myrsinaceae } & \text { A } & \text { N } \\ \text { Ericaceae } & \text { Ar } & \text { E } \\ \text { Anacardiaceae } & \text { A } & \text { E } \\ \text { Fabaceae } & \text { A } & \text { E } \\ \text { Annonaceae } & \text { A } & \text { NC } \\ \text { Proteaceae } & \text { A } & \text { NC } \\ \text { Arecaceae } & \text { A } & \text { E } \\ \text { Salicaceae } & \text { A } & \text { E } \\ \text { Caprifoliaceae } & \text { A } & \text { E } \\ & & \end{array}$

AV

N

$\mathrm{NZ}$, As, Ca, A, T

AV

$\mathrm{Ca}, \mathrm{Ar}, \mathrm{T}$

$A V, A B$

E

(1)

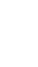

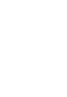

A, As, CGW, IP, J, Ma, NZ, Se, T AV, AB Ar, As, Br, GCW, E, EUA, IC, IG, IP, J, AV, AB NZ, T

casca
frutos

$T, E U A$

AV

$A, A r, T$

$\operatorname{Ar}, \mathrm{T}$

AV

$A V, A B$

AV

sementes, folha A, Ar, As, Ca, E, EUA, Fr, G, Hu, J, NZ, R, AV

$\begin{array}{ll}\text { sementes, folha, } & \text { P, Po, S, U, T } \\ \text { casca } & \end{array}$

NC $\quad A V, A B$

$\mathrm{NC} \quad \mathrm{AV}, \mathrm{AB}$

AV

$R, A V$

frutos (em $\quad A V, A B$

quantidade),

seiva (irritante

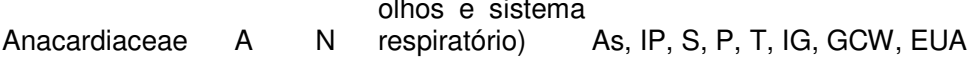

Anacardiaceae A NC fruto e seiva A, As, GCW, EUA, IC, IP, Ma, NZ, P, T AV, AB

$\begin{array}{llll}\text { Fabaceae } & \mathrm{A} N \mathrm{~N}, \mathrm{IC} & \mathrm{AV}, \mathrm{AB}\end{array}$

$\begin{array}{llll}\text { Euphorbiaceae } & A \quad N C & A V, A B\end{array}$ 
144 Senna bicapsularis (L.) Roxb. Senna macranthera (DC. ex Collad.) H.S. Irwin \& 145 Barneby

146 Senna multijuga (Rich.) H.S. Irwin \& Barneby

147 Senna spectabilis (DC.) H.S. Irwin \& Barneby

148 Sesbania virgata (Cav.) Pers.

149 Spartium junceum L.

150 Spiraea cantoniensis Lour.

151 Syagrus romanzoffiana (Cham.) Glassman

152 Tabebuia alba (Cham.) Sandwith

153 Tabebuia chrysotricha (Mart. ex A. DC.) Standl.

154 Tabebuia heptaphylla (Vell.) Toledo

155 Tabebuia roseo-alba (Ridl.) Sandwith

156 Tabebuia umbellata (Sond.) Sandwith

157 Taxodium distichum (L.) Rich.

158 Thuja occidentalis L.

159 Tibouchina sellowiana Cogn.

160 Tipuana tipu (Benth.) Kuntze

161 Vitex montevidensis Cham.

162 Vochysia tucanorum Mart.

163 Weigela florida (Bunge) A. DC

164 Wisteria floribunda (Willd.) DC

165 Zanthoxylum rhoifolium Lam.

LEGENDA:

F.V. = forma de vida: $A=$ arbóreo, $B=$ arbustivo, $H=$ herbáceo, $T=$ trepadeira;

$P .=$ procedência: $\mathrm{N}=$ nativa do Brasil, $\mathrm{NC}=$ nativa em Curitiba; $\mathrm{E}=$ exótica no Brasil;

TÓXICA = parte da planta que apresenta princípios tóxicos:

INVASORA = país em que a espécie é considerada invasora: $\mathrm{A}=$ Austrália; $\mathrm{Ar}=$ Argentina; $\mathrm{As}=$ África do $\mathrm{Sul} ; \mathrm{Br}=\mathrm{Brasil} ; \mathrm{Bo}=\mathrm{Botwana} ; \mathrm{Ca}=\mathrm{Canadá}$, $\mathrm{C}=\mathrm{China}$; $=\mathrm{Espanha;} \mathrm{EUA}=\mathrm{Estados}$ Unidos; $G$ = Alemanha; IC = Ilhas do Caribe; IG = Ilha de Galápagos - Equador; IP = Ilhas do Pacífico; Hu = Hungria; Ma = Malawii; Na = Namíbia; NZ = Nova Zelândia; Pa = Paquistão; P = Portugal; $\mathrm{Po}=$ Polônia; U = Ucrânia; $\mathrm{Se}=$ Seychelles; $\mathrm{S}=$ Suíça; GCW = The Global Compendium of Weeds; $\mathrm{T}$ = trópicos e subtrópicos;

USOS =destino das mudas produzidas: $R=$ arborização de ruas; $A V=$ áreas verdes; $A B=$ adensamento de bosques.
As, IP, IG

IC, T

$R, A V, A B$

$R, A V, A B$

$A V, A B$

$A V, A B$

AV

$R, A V, A B$

$R, A V, A B$

$R, A V, A B$

$R, A V, A B$

$A V, A B$

$A V, A B$

AV

$R, A V, A B$

As, Ma

$R, A V, A B$

$A V, A B$

$A V, A B$

AV

AV

$A V, A B$
Fabaceae $\quad T \quad E$ 


\section{CONCLUSÕES}

A partir da lista de espécies considerada, constatou-se, entre espécies produzidas no Horto Municipal da Barreirinha, a presença significativa de espécies inadequadas aos usos propostos pelo mesmo, por apresentarem princípios tóxicos e/ou alérgicos, que são aspectos muito perigosos à saúde humana. No que se refere aos aspectos ecológicos, ainda são produzidas espécies exóticas e plantas invasoras, que podem prejudicar a biodiversidade local.

Devido à grande responsabilidade deste Horto no fomento de vegetação, recomenda-se a adoção desta análise para estabelecer critérios para a seleção e produção de mudas de espécies adequadas para as áreas públicas da cidade de Curitiba.

\section{REFERÊNCIAS}

AGRA, M. F.; FREITAS, P. F.; BARBOSA-FILHO, J. M. Synopsis of the plants known as medicinal and poisonous in Northeast of Brazil. Rev. Bras. Farmacogn., João Pessoa, v. 17 , n. 1 , p. 114-140, 2007.

ALBUQUERQUE, J. M. Plantas tóxicas: no jardim e no campo. Belém: FCAP. Serviço de Documentação e Informação, 1980. 120 p.

BARROSO, C. M.; DELWING, A. B.; KLEIN, G. N.; BARROS, I. B. I.; FRANKE, L. B. Considerações sobre a propagação e o uso ornamental de plantas raras ou ameaçadas de extinção no Rio Grande do Sul. Rev. Bras. Agroecologia, Porto Alegre, v. 2, n. 1, fev. 2007.

BINGGELI, P.; HALL, J.B.; HEALEY, J.R. An overview of invasive woody plants in the tropics. Bangor: University of Wales/School of Agricultural and Forest Sciences, 1998. 83 p.

BIONDI, D.; ALTHAUS. M. Árvores de rua de Curitiba: cultivo e manejo. Curitiba: FUPEF, 2005. $182 \mathrm{p}$.

BIONDI, D.; KISCHLAT, E. A vegetação urbana e a biodiversidade. Diálogo, Canoas, n. 1, p. 155-168, 2006.

BIONDI, D.; MACEDO, J. H. P. Plantas invasoras encontradas na área urbana de Curitiba PR. Floresta, Curitiba, v. 38, n.1, p. 129-144, 2008. 
BIONDI, D.; LEAL, L; SCHAFFER, M. Aspectos importantes das plantas ornamentais em escolas públicas estaduais da cidade de Curitiba/PR. Rev. Bras. Ciênc. Agrár., Recife, v. 3, 2008. No prelo.

BLACKWELL, W. H. Poisonous and medicinal plants. New Jersey: Prentice Hall, 1990. $329 \mathrm{p}$.

BURT, J. W.; MUIR, A. A.; PIOVIA-SCOTT, J.; VEBLEN, K. E.; CHANG, A. L.; GROSSMANN, J. D., WEISKEL, H. W. Preventing horticultural introductions of invasive plants: potential efficacy of voluntaries initiatives. Biol. Invasions, v. 9, p. 909-923, 2007.

CAVALCANTI, M. F. C.; DANTAS, I. C.; LIRA, R. S.; OLIVEIRA, J. M. C.; ALBUQUERQUE, H. N.; ALBUQUERQUE, I. C. S. Identificação dos vegetais tóxicos da cidade de Campina Grande-PB. Revista de Biologia e Ciências da Terra, Campina Grande, v. 3, n. 1, p. 1-19, 2003.

CESP. Guia de arborização. 3.ed. São Paulo: CESP, 1988. 33 p. (Coleção Ecossistemas Terrestres, 006).

DE-KNEGT, L. V.; SILVA, J. A.; MOREIRA, E. C.; SALES, G. L. Morcegos capturados no município de Belo Horizonte. Arq. Bras. Med. Vet. Zootec., Belo Horizonte, v. 57, n. 5, p. 576-583, 2005.

FIOCRUZ. Plantas tóxicas no Brasil. Série Prevenindo Intoxicações. Julho, 2001 (cartaz e folder)

FROHNE, D.; PFÄNDER, H.J. A colour atlas of poisonous plants: a handbook for pharmacists, doctors, toxicologists and biologists. London: Wolfe, 1983. 291p.

GCW. A Global Compendium of Weeds. Disponível em: <http://www.hear.org/gcw/html/index.html> Acesso em: 01 maio 2007.

GONÇALVES, E. O.; PAIVA, H. N.; GONÇALVES, W.; JACOVINE, L. A. G. Diagnóstico dos viveiros municipais no Estado de Minas Gerais. Ciência Florestal, Santa Maria, v. 14, n. 2, p. 1-12, 2004. 
HENDERSON, L. Alien weeds and invasive plants: a complete guide to declared weeds and invaders in South Africa. Cape Town: Plant Protection Research Institute Agricultural Research Council, 2001. 300 p.

HORUS INSTITUTE. Espécies exóticas invasoras. Disponível em: <http://tncweeds.ucdavis.edu/global/brasil/horuslist.html> Acesso em: 01 maio 2007.

INVASIVE PLANTS DATABASE. Invasive Plants Database (IPDB). Disponível em: <http://invasive.m-fuukei.jp/rlist.php> Acesso em: 01 maio 2007.

JARDIM DE FLORES. Plantas tóxicas: perigo no jardim. Disponível em: <http://www.jardimdeflores.com.br/PAISAGISMO/A20plantastoxicas.htm> Acesso em: 10 junho 2007.

LEAL, L. Custos das árvores de rua - Estudo de caso: cidade de Curitiba/PR. Curitiba, 2007. 115 f. Dissertação (Mestrado em Engenharia Florestal) - Setor de Ciências Agrárias, Universidade Federal do Paraná.

LORENZI, H.; MATTOS, F. J. A. Plantas medicinais no Brasil: nativas e exóticas cultivadas. Nova Odessa: Instituto Plantarum, 2002. 512p.

LUCAS, G. N. Plant poisoning: a hospital-based study in Sri Lanka. Indian J. Pediatr., New Delhi, n. 64, p. 495-502, 1997.

MACK, R. N.; SIMBERLOFF, D.; LONSDALE, W. M.; EVANS, H.; CLOUT, M.; BAZZAA, F. A. Biotic invasions: causes, epidemiology, global consequences and control. Ecol. Appl., n. 10, p. 689-710, 2000.

PARANÁ. Portaria IAP $n^{\circ}$ 074, de 19 de abril de 2007. Reconhece a Lista Oficial de Espécies Exóticas Invasoras para o Estado do Paraná, estabelece normas de controle e dá outras providências.

PREFEITURA MUNICIPAL DE CURITIBA. Horto Barreirinha. Disponível em: $<$ http://www.curitiba.pr.gov.br/Secretaria.aspx?idf=393\&servico=26>Acesso em: 27 fevereiro 2008. 
REICHARD, S. H.; WHITE, P. Horticulture as a pathway of invasive plant introduction in the United States. BioScience, Washington, v. 51, n. 2, p. 103-113, 2001.

REIS, A.; ANJOS, A.; LESSA, A. P.; BECHARA, F. C. Critérios para a seleção de espécies na arborização urbana ecológica. Sellowia, Itajaí, v. 53-55, p. 51-67, 2003.

SANTOS, N. R. Z.; TEIXEIRA, I. F. Arborização de vias públicas: ambiente x vegetação. Santa Cruz do Sul: Instituto Souza Cruz, 2001. 135 p.

SWEARINGEN, J. WeedUS: Database of Plants Invading Natural Areas in the United States. Disponível em: <http://www.nps.gov/plants/alien/list/WeedUS.xls> Acesso em: 10 maio 2007.

ZILLER, S. R.; ZALBA, S. Propostas de ação para prevenção e controle de espécies exóticas invasoras. Natureza \& Conservação, Curitiba, v. 5, n. 2, p. 8-15, 2007. 